\title{
FIRST EVIDENCE OF DUSTY DISKS AROUND HERBIG Be STARS
}

\author{
A. Fuente, ${ }^{1}$ A. Rodríguez-Franco,${ }^{2,3}$ L. Testi, ${ }^{4}$ A. Natta, ${ }^{4}$ R. Bachiller, ${ }^{1}$ And R. Neri ${ }^{5}$ \\ Received 2003 July 9; accepted 2003 October 1; published 2003 November 3
}

\begin{abstract}
We have carried out a high-sensitivity search for circumstellar disks around Herbig Be stars in the continuum at 1.4 and $2.7 \mathrm{~mm}$ using the IRAM interferometer at the Plateau de Bure. In this Letter, we report data on three well-studied B0 stars: MWC 1080, MWC 137, and R Mon. The two latter have also been observed in the continuum at 0.7 and $1.3 \mathrm{~cm}$ using the NRAO Very Large Array. We report the detection of circumstellar disks around MWC 1080 and R Mon with masses of $M_{d} \sim 0.003$ and $0.01 M_{\odot}$, respectively, while for MWC 137 we estimate a disk mass upper limit of $0.007 M_{\odot}$. Our results show that the ratio $M_{d} / M_{*}$ is at least an order of magnitude lower in Herbig Be stars than in Herbig Ae and T Tauri stars.

Subject headings: circumstellar matter — radio continuum: stars — stars: individual (MWC 137, MWC 1080, R Monocerotis) — stars: pre-main-sequence
\end{abstract}

\section{INTRODUCTION}

The existence of accretion disks around massive stars $\left(M>5 M_{\odot}\right)$ remains a matter of debate. There is increasing evidence for the existence of flattened structures (disks) around high-mass protostars. However, there is no clear evidence of disks in later phases, namely, in Herbig Be (HBe) stars. Natta, Grinin, \& Mannings (2000) compiled the interferometric observations in millimeter continuum around $\mathrm{HBe}$ stars and found that the occurrence of disks in HBe stars is $\sim 0$. This contrasts with the case of the lower mass HAe stars $\left(2 M_{\odot} \leq M_{*} \leq 5 M_{\odot}\right)$ in which the occurrence of circumstellar disks is similar to that in T Tauri stars (TTSs). They propose that the lack of disks around $\mathrm{HBe}$ stars is due to the rapid evolution of these objects, which disperse the surrounding dust and gas in about $10^{6} \mathrm{yr}$ (Fuente et al. 2002). However, this lack of detection can be a sensitivity effect. $\mathrm{HBe}$ stars are usually further away than HAe stars, and higher sensitivity interferometric observations are required to detect circumstellar disks around these objects. In this Letter, we present the first results of a high-sensitivity search for circumstellar disks around three B0 stars, MWC 1080, MWC 137, and R Mon, which are the best studied of the five Herbig B0 stars in the northern sky listed by Thé, de Winter, \& Pérez (1994).

\section{OBSERVATIONS}

Interferometric observations in the continuum at 1.4 and $2.7 \mathrm{~mm}$ have been carried out toward the Herbig Be stars MWC 1080, MWC 137, and R Mon, using the IRAM ${ }^{6}$ array at Plateau de Bure (PdB), France, in the $\mathrm{CD}$ set of configurations. MWC 137 and $\mathrm{R}$ Mon were also observed with the $\mathrm{NRAO}^{7}$ Very Large Array (VLA) at 0.7 and $1.3 \mathrm{~cm}$ in its D configuration. Flux calibration is accurate within $10 \%$ at $2.7 \mathrm{~mm}$ and $20 \%$

\footnotetext{
${ }^{1}$ Observatorio Astronómico Nacional, Apartado 1143, E-28800 Alcalá de Henares, Spain; a.fuente@oan.es.

${ }^{2}$ Departamento Matemática Aplicada (Biomatemática), Universidad Complutense de Madrid, Avenida Arcos de Jalón s/n, E-28037 Madrid, Spain.

${ }^{3}$ Consejo Superior de Investigaciones Científicas, IEM, DAMIR, Serrano 121, E-28006 Madrid, Spain.

${ }^{4}$ Osservatorio Astrofisico di Arcetri, Largo Enrico Fermi 5, I-50125 Firenze, Italy.

${ }^{5}$ Institut de Radioastronomie Millimétrique, 300 rue de la Piscine, 38406 St. Martin d'Hères Cedex, France.

${ }^{6}$ IRAM is supported by INSU/CNRS (France), MPG (Germany), and IGN (Spain).

${ }^{7}$ The NRAO is a facility of the National Science Foundation operated under cooperative agreement by Associated Universities, Inc.
}

at $1.4 \mathrm{~mm}$ in the $\mathrm{PdB}$ interferometer images and within $20 \%$ in the VLA images. No correction for primary beam attenuation has been applied. PdB interferometer and VLA images are shown in Figure 1. Flux densities at the star positions are shown in Table 1.

\section{MWC 1080}

Although we have not observed MWC 1080 at centimeter wavelengths, some information about the centimeter emission can be found in the literature. Curiel et al. (1989), from 10" resolution observations at $6 \mathrm{~cm}$, detected a weak extended component that includes the star and the reflection nebula. Skinner, Brown, \& Stewart (1993), with higher angular resolution $\left(\sim 1^{\prime \prime}\right)$, tentatively detected at $3.6 \mathrm{~cm}$ a compact source at the star position. Recently, Girart et al. (2002) detected three $6 \mathrm{~cm}$ sources using the VLA with an angular resolution of $\sim 5^{\prime \prime}$. One of these sources is coincident with the star position. They did not detect a counterpart of this emission at $2.0 \mathrm{~cm}$ with an upper limit of $\leq 0.32 \mathrm{mJy}$.

Intense emission is detected in the millimeter continuum images observed with the PdB interferometer (see Figs. $1 a$ and $1 b$ ). Interferometric observations at $2.7 \mathrm{~mm}$ in MWC 1080 were previously reported by Di Francesco et al. (1997) with a nondetection. Our image improves by a factor of 10 the sensitivity and by a factor of 2 the angular resolution of their data. The millimeter continuum emission in MWC 1080 is extended. Two intense components connected by a bridge of weak emission are observed in the $2.7 \mathrm{~mm}$ image. These components are much closer to the star than the VLA $6 \mathrm{~cm}$ sources detected by Girart et al. (2002). We will refer to these components as "NW" and "SE." The better angular resolution of the $1.4 \mathrm{~mm}$ image resolves the NW and SE clumps in several components: "NW1," "SE1," "SE2," and "Star." The total flux in the $1.4 \mathrm{~mm}$ image is $\sim 24 \mathrm{mJy}$. In our single-dish data, we measured a $1.4 \mathrm{~mm}$ flux of $73.8 \mathrm{mJy}$ at the star position with an angular resolution of $\sim 10$ ".5 (Fuente et al. 1998). Thus, the interferometer has resolved the $1.4 \mathrm{~mm}$ emission and missed $\sim 70 \%$ of the flux. The Star component is coincident with the optical star position and remains unresolved in the $1.4 \mathrm{~mm}$ image. This implies that its size is less than 2", which corresponds to a spatial extent of less than $2000 \mathrm{AU}$ at the distance of MWC $1080(d=1000 \mathrm{pc})$. This size is compatible with the emission arising in a circumstellar disk. 

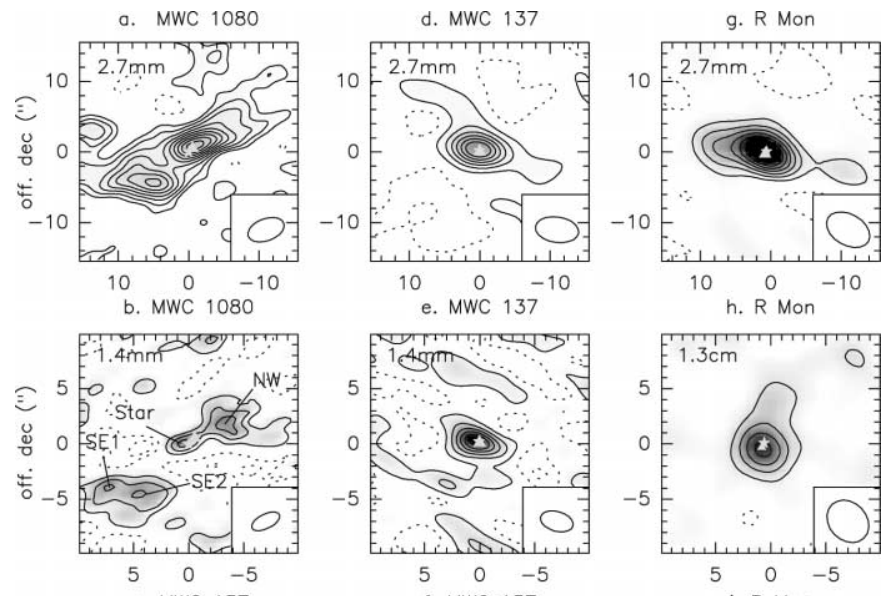

c. MWC 137
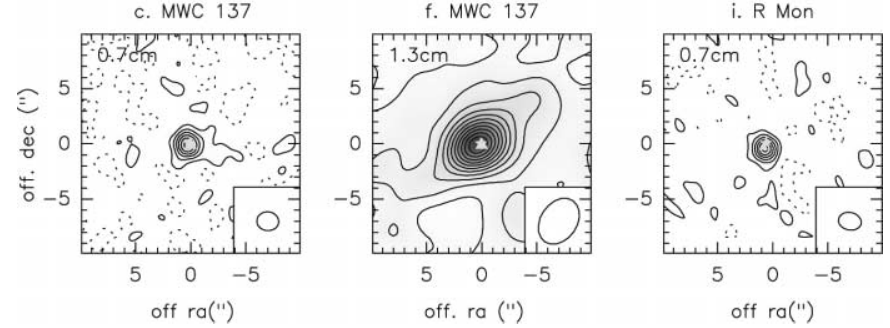

FIG. 1.-Continuum images obtained with the PdB and VLA arrays. The star marks the optical position (MWC 1080: $23^{\mathrm{h}} 17^{\mathrm{m}} 25^{\mathrm{s}} .574,60^{\circ} 50^{\prime} 43^{\prime \prime} .34$; MWC 137: $06^{\mathrm{h}} 18^{\mathrm{m}} 45^{\mathrm{s}} .504,+15^{\circ} 16^{\prime} 52^{\prime \prime} \cdot 4$; R Mon: $06^{\mathrm{h}} 39^{\mathrm{m}} 09^{\mathrm{s}} \cdot 94,+08^{\circ} 44^{\prime} 10^{\prime \prime} 0$ ), and the filled triangle the position of the radio source (Skinner et al. 1993). Contour levels are (a) -2.0 to -0.2 and $0.2-4.0 \mathrm{mJy}^{-1}$ beam $^{-1}$ by $0.2 \mathrm{mJy}^{-1}$ beam $^{-1},(b)-1.6,-0.7$, $0.7-3.4 \mathrm{mJy}^{-1}$ beam ${ }^{-1}$ by $0.9 \mathrm{mJy}^{-1} \mathrm{bem}^{-1},(c)-0.2 \mathrm{mJy}$ beam $^{-1}, 0.2-2.2$

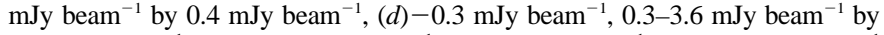
$0.6 \mathrm{mJy}_{\text {beam }}^{-1},(e)-1.8 \mathrm{mJy}^{\text {beam }}{ }^{-1},-0.6 \mathrm{mJy}^{-1}$ beam $^{-1}, 0.6-6.6 \mathrm{mJy}^{-1}$ beam $^{-1}$ by $1.2 \mathrm{mJy}^{-1}$ beam $^{-1}$; $(f)-0.2 \mathrm{mJy}$ beam $^{-1}, 0.2-2.4 \mathrm{mJy}^{-1}$ beam $^{-1}$ by

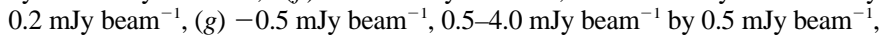
(h) $-0.2 \mathrm{mJy}^{-1}$ beam $^{-1}, 0.2-0.8 \mathrm{mJy}$ beam ${ }^{-1}$ by $0.2 \mathrm{mJy}^{-1}$ beam $^{-1}$, (i) -0.2 mJy beam ${ }^{-1}, 0.2-1.4$ mJy beam $^{-1}$ by $0.2 \mathrm{mJy}_{\text {beam }}{ }^{-1}$.

\section{MWC 137}

MWC 137 is associated with the $1^{\prime}$ size $\mathrm{H}$ II region S266 (Fich 1993). Observations of this region at $3.6 \mathrm{~cm}$ were reported by Skinner et al. (1993). Our $1.3 \mathrm{~cm}$ image of MWC 137 consists of a compact source coincident with the HBe star surrounded by a thin shell with a radius of $30^{\prime \prime}-40^{\prime \prime}$ from the point source. Weak emission is found toward the thin shell and the point source. In the $0.7 \mathrm{~cm}$ image, the compact source appears more intense while the emission of the thin shell becomes fainter. Since our aim is the detection of a circumstellar disk, we are interested in only the compact component (see Table 1).

The continuum millimeter images show intense emission centered at the star position. The total flux in the $1.4 \mathrm{~mm}$ image is $\sim 10 \mathrm{mJy}$, i.e., $\sim 30 \%$ of the flux measured in the single-dish observations (Fuente et al. 1998). From our $1.4 \mathrm{~mm}$ image, we derive a source size of $1^{\prime \prime} .8 \times 0.8$, which corresponds to $\approx 2300 \times 1000 \mathrm{AU}$ assuming a distance of $1300 \mathrm{pc}$ and a position angle of $75^{\circ} \pm 15^{\circ}$. Note that the accuracy of the position angle estimate is heavily limited by the signal-to-noise ratio of the data.

\section{R MON}

R Mon was observed at 6, 3.6, and $2 \mathrm{~cm}$ using the VLA by Skinner et al. (1993). They detected continuum emission at a position slightly shifted from the optical one. A compact source is detected in $\mathrm{R}$ Mon at 1.3 and $0.7 \mathrm{~cm}$. The position of this source is offset $(0.74,-0.45)$ from the optical position but coincident with the radio source. A jetlike feature is observed in the $1.3 \mathrm{~cm}$ image, which is surely driven by the radio source. This jet was already detected by Brugel, Mundt, \& Buhrke (1984) in optical lines. Since the uncertainty of the optical position is not known, the identification of the radio source with $\mathrm{R}$ Mon is uncertain.

We have detected continuum emission toward the star in the $2.7 \mathrm{~mm}$ image with a peak emission of $4.1 \mathrm{mJy}^{\text {beam }}{ }^{-1}$. The total integrated flux is $6.4 \mathrm{mJy}$, revealing that the emission is extended. Deconvolving the $2.7 \mathrm{~mm}$ image with the beam, we estimate an emission size of $3^{\prime \prime}-4$ ", which corresponds to $\approx 3000 \mathrm{AU}$ at the distance of $800 \mathrm{pc}$. Natta et al. (2000) reported a total $2.7 \mathrm{~mm}$ flux of $13.0 \pm 1.3 \mathrm{mJy}$ toward R Mon based on data by V. Mannings (1998, unpublished). This flux exceeds by a factor of 2 our present result. This reinforces our conclusion that the emission is extended. Then, the different beams and calibration uncertainty can account for this discrepancy. The $2.7 \mathrm{~mm}$ continuum emission is elongated in the direction perpendicular to the bipolar nebula axis, and the centroid of the emission lies to the northeast of the position of the radio source. The shape and position of the $2.7 \mathrm{~mm}$ emission suggest that the $2.7 \mathrm{~mm}$ emission is tracing a different emission component from that traced by the emission at centimeter wavelengths and argues in favor of the existence of a flattened structure (disk) around the star.

\section{SPECTRAL ENERGY DISTRIBUTIONS}

The spectral energy distributions (SEDs) in the centimeter to millimeter range of MWC 1080, MWC 137, and R Mon are shown in Figure 2. In order to avoid the contribution of the envelopes of these sources to the continuum emission, only interferometric measurements have been included in these SEDs. Even though, some problems still remain. The emission at centimeter wavelengths in MWC 1080 is extended. The negative index derived by comparing the flux at 6 and $3.6 \mathrm{~cm}$ is very likely due to the larger beam of the $6 \mathrm{~cm}$ flux $\left(5^{\prime \prime} .5 \times 55^{\prime \prime} .03\right)$ compared with that of the $3.6 \mathrm{~cm}$ one $(1 " .24 \times 0.83)$. The disk emission should be unresolved at the distance of our source. Therefore, we are not interested in the extended component and consider the $6 \mathrm{~cm}$ flux as an upper limit. Unfortunately, the freefree emission arising in the stellar wind is also expected to be unresolved with our beam and could make an important contri-

TABLE 1

OBSERVED FLuXes

\begin{tabular}{ccccc}
\hline \hline Object & $\begin{array}{c}S_{1.3 \mathrm{~cm}} \\
\left(\mathrm{mJy} \mathrm{beam}^{-1}\right)\end{array}$ & $\begin{array}{c}S_{0.7 \mathrm{~cm}} \\
\left(\mathrm{mJy} \mathrm{beam}^{-1}\right)\end{array}$ & $\begin{array}{c}S_{2.7 \mathrm{~mm}} \\
\left(\mathrm{mJy} \mathrm{beam}^{-1}\right)\end{array}$ & $\begin{array}{c}S_{1.4 \mathrm{~mm}} \\
\left(\mathrm{mJy} \mathrm{beam}^{-1}\right)\end{array}$ \\
\hline MWC 1080 $\ldots \ldots$ & $\ldots$ & $\ldots$ & $<1.7$ & $3.1(0.2)$ \\
MWC 137 $\ldots \ldots$. & $1.5(0.5)^{\mathrm{a}}$ & $2.35(0.13)$ & $4.1(0.2)$ & $7.1(0.6)$ \\
R Mon $\ldots \ldots \ldots \ldots$ & $0.78(0.10)$ & $1.26(0.13)$ & $4.1(0.5)$ & $<15$ \\
\hline
\end{tabular}

a Subtracting the emission of the extended component. 

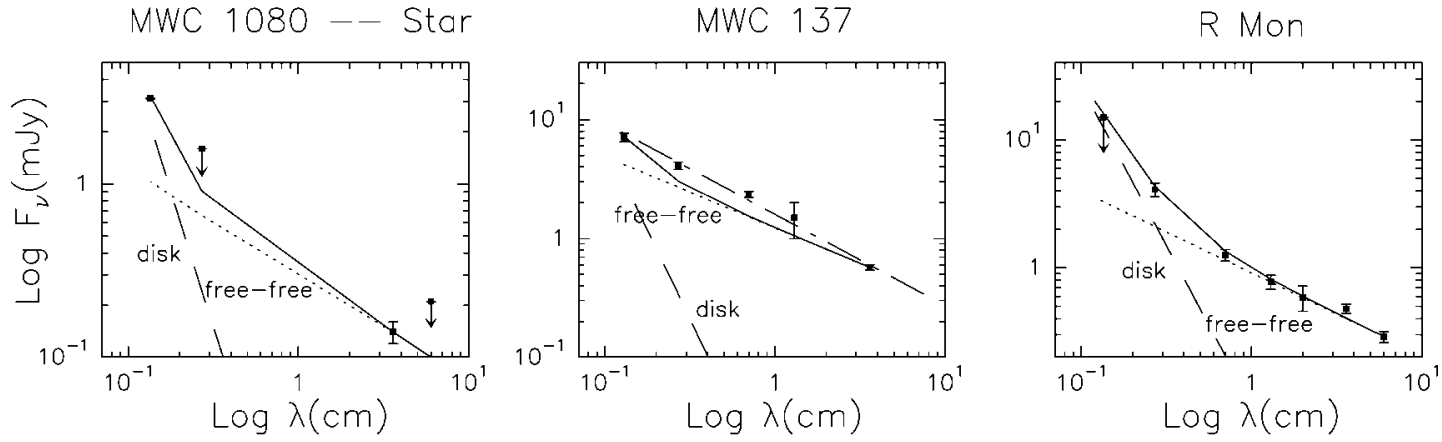

FIG. 2.- SEDs at centimeter and millimeter wavelengths of MWC 1080, MWC 137, and R Mon. The fluxes at centimeter wavelengths have been taken from Skinner et al. (1993) and Girart et al. (2002) and at millimeter wavelengths from this Letter. The short-dashed lines are model predictions for the free-free emission component. The long-dashed lines are model predictions for circumstellar disks of $0.003 M_{\odot}$ and $\beta=1$ (MWC 1080), $0.007 M_{\odot}$ and $\beta=1$ (MWC 137), and $0.01 M_{\odot}$ and $\beta=0.5$ (R Mon). The solid line is the sum of both components. The short-dash-long-dashed straight line in the central panel is the predicted fluxes for the emission arising in a stellar wind with $\alpha=0.76$ in MWC 137.

bution to the continuum flux even at millimeter wavelengths. In order to derive the disk mass in MWC 1080, we have assumed that the $3.6 \mathrm{~cm}$ flux is due to free-free wind emission and subtracted it from the observed millimeter fluxes. These corrected values are then interpreted as optically thin disk emission, according to the expression $F_{\lambda}=d^{-2} M_{d} \kappa_{\lambda} B_{\lambda}\left(T_{d}\right)$, with $\kappa=$ $0.01[\lambda(\mathrm{mm}) / 1.3]^{-\beta} \mathrm{cm}^{-2} \mathrm{~g}^{-1}$. We have assumed a constant value of $T_{d}=215 \mathrm{~K}$ following the recommendation of Natta et al. (2000) for B0 stars. With these assumptions, we obtain from the $1.4 \mathrm{~mm}$ flux a disk mass of $0.003 M_{\odot}$. Since we have only an upper limit to the $2.7 \mathrm{~mm}$ flux, the value of $\beta$ cannot be determined. We have compared the disk mass derived with this simplified expression to those of more elaborated models (see Dullemond, van Zadelhoff, \& Natta 2002), varying the disk outer radius and the surface density profile over a large range of possible values, and confirmed the conclusion of Natta et al. (2000) that the values of $M_{d}$ derived in this way are accurate within a factor of $2-3$. We have also considered the possibility that the $3.6 \mathrm{~cm}$ emission arises in an optically thin $\mathrm{H}$ II region instead of free-free emission. With only two points in the SED, we cannot discern between these two possibilities. In this case, we obtain a slightly larger value of the disk mass of $M_{d} \sim 0.004 M_{\odot}$. Therefore, we estimate that the mass of the disk around MWC 1080 is $0.003 \pm 0.001 M_{\odot}$.

The complete SED of MWC 137 can be fitted with a single component with spectral index $\alpha=0.76 \pm 0.01$. This spectral index is consistent with that expected in the free-free emission arising in the stellar wind. Although a value of $\alpha=0.6$ is expected for an ionized isotropic wind, small deviations of this value can be explained by a different geometry or a partially ionized wind. However, we cannot discard the existence of a very low mass disk. In order to estimate an upper limit to the disk mass, we have extrapolated the $3.6 \mathrm{~cm}$ flux to millimeter wavelengths with an spectral index of 0.6. Then, we have estimated the disk mass from the excess flux at $1.4 \mathrm{~mm}$. With the same values of $\kappa$ and $T_{d}$ as in the case of MWC 1080, we obtained an upper limit to the disk mass of $0.007 M_{\odot}$.

The SED of R Mon is the most complete one. We have been able to fit all the centimeter data using a spectral index, $\alpha_{\mathrm{cm}} \approx 0.64$, which is characteristic of a spherical stellar wind. In order to estimate the mass of the circumstellar disk, we have extrapolated the free-free emission to millimeter wavelengths and fitted the millimeter part of the SED with a simple disk model. As in the case of MWC 1080, we have assumed an unresolved disk at a constant temperature, $T_{d}=215 \mathrm{~K}$, and adjusted the values of $M_{d}$ and $\beta$. Only values of $\beta=$ $0.25-0.5$ fit our observational points. A value of $\beta$ larger than 0.5 would produce an excess of flux at $1.4 \mathrm{~mm}$. This low value of $\beta$ could be interpreted as an optically thick compact disk or an optically thin disk with large grains. The first possibility would imply millimeter fluxes larger than those measured. A small disk of $R=100 \mathrm{AU}$ should have a mass of $0.35 M_{\odot}$ in order to have $\tau_{1.4 \mathrm{~mm}} \sim 1$. But in this case, the $1.4 \mathrm{~mm}$ flux would be at least an order of magnitude larger than our upper limit. The situation would be worse for a larger disk. The second possibility seems more plausible. Similarly low values of $\beta$ have been found in Herbig Ae and T Tauri disks and reveal highly processed dust (Beckwith \& Sargent 1991; Testi et al. 2003). With the fiducial value of the dust emissivity, we derive a disk mass of $0.01 M_{\odot}$. Note, however, that if the low value of $\beta$ is interpreted as evidence of grain growth, one needs to reduce the fiducial $2.7 \mathrm{~mm}$ dust emissivity by a factor of about 4 (Testi et al. 2003) and to increase the estimated disk mass by a similar amount.

\section{DISK DETECTIONS IN HBe STARS}

Our results prove the existence of circumstellar dust around MWC 1080 and R Mon. However, it is very difficult to interpret it beyond doubt as evidence of a circumstellar disk. In general, a compact and small $1.4 \mathrm{~mm}$ source $\left(<1^{\prime \prime}-2^{\prime \prime}\right)$ at the star position is considered as a disk detection. This criterion seems sufficient in TTSs located at distances of $\sim 150 \mathrm{pc}$, in which an angular resolution of 1" corresponds to a linear scale of 100-200 AU, the typical size of circumstellar disks. Since HBe stars are usually located at distances larger than $1 \mathrm{kpc}$, even the higher angular resolution provided by interferometers, $\sim 1^{\prime \prime}$, corresponds to sizes of a few thousands of $\mathrm{AU}$, and it is not possible to distinguish between "bona fide" circumstellar disks and a flattened structure of a few thousand AU.

Another difficulty comes from the fact that many HBe stars are likely associated with lower mass companions that will not be resolved in our beam. MWC 1080 is a close binary with a separation of 0"7 (Leinert, Richichi, \& Haas 1997). Midinfrared observations by Polomski et al. (2002) using the Keck II telescope resolve the binary and show that warm material $(\sim 350 \mathrm{~K})$, with a $3-10 \mu \mathrm{m}$ spectral index consistent with the existence of a circumstellar disk, is surrounding MWC 1080A. 
A cooler dust component is associated with MWC 1080B. We have detected an unresolved millimeter continuum source in MWC 1080. We consider that this source is a good candidate for disk detection around an $\mathrm{HBe}$ star. However, since the angular resolution of our observations is not sufficient to resolve the two binary components, we cannot exclude the possibility that the emission is associated with the younger companion.

$\mathrm{R}$ Mon is also a close binary with a separation of 0 ".69. Its companion is a very young TTS. Close et al. (1997) found an extinction of $A_{v}=13.1 \mathrm{mag}$ toward the star, which they interpreted as due to an optically thick disk of $R=100$ AU. Our $2.7 \mathrm{~mm}$ image reveals the existence of a compact source of $\approx 3000 \times 2300 \mathrm{AU}$. This size is larger than that expected for a circumstellar disk and suggests the existence of a flattened envelope surrounding the disk. Our value of the disk mass may be overestimated owing to the contribution of the flattened envelope. However, the analysis of the millimeter spectral index shows that the grains responsible for the emission are heavily processed, so that most of the emission is very likely arising in a circumstellar disk. If this is true, it is unlikely that the disk is associated with the TTS companion, since in this case one should use in deriving the disk mass a value of $T_{d} \sim 15 \mathrm{~K}$ (Natta et al. 2000) and the estimated mass would increase to values in the range $0.2-0.5 M_{\odot}$, too large for a TTS disk.

We have completed the compilation of (interferometric) disk observations of HAeBe stars carried out by Natta et al. (2000) with the observations in MWC 1080, MWC 137, and R Mon reported in this Letter, and the observations in HD 200775 and $\mathrm{LkH} \alpha 234$ reported by Fuente et al. (2001). In Figure 3, we plot the disk masses as a function of the spectral type of the star and the stellar age. We have plotted MWC 1080 and R Mon as disk detections; however, as discussed in $\S 6$, it is possible that the "disk" emission is either overestimated or not associated with the Herbig Be star itself but with unresolved companions, thus reinforcing our conclusions. In any case, disk masses in $\mathrm{HBe}$ stars are at least an order of magnitude lower than in HAe stars. Plotting $M_{d} / M_{*}$ as a function of the spectral type, one finds that while the value of $M_{d} / M_{*}$ is roughly constant and equal to 0.04 for stars with spectral type A0-M7, $M_{d} / M_{*}<0.001$ in HBe stars. The same effect remains when one plots the disk mass against the stellar age. The values of disk masses in HBe stars are systematically lower than in HAe stars for stars with ages between $10^{5}$ and $10^{7} \mathrm{yr}$. This lack of massive disks in HBe stars even for stars as young as $10^{5} \mathrm{yr}$ puts several constraints on the possibility of forming planetary systems around massive stars. In a timescale of less than $10^{6} \mathrm{yr}$, the disk mass around an HBe star is similar to or even lower than the total mass of the planets in the solar
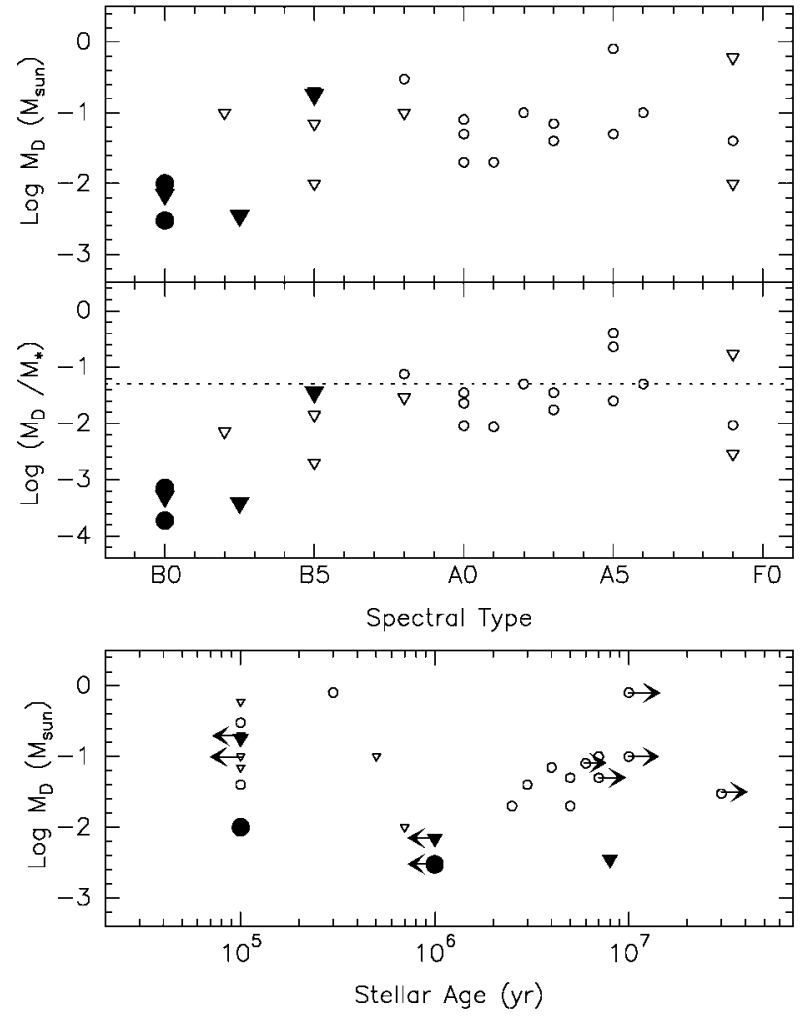

FIG. 3.-Plot of the $M_{d}$ and $M_{d} / M_{*}$ vs. the spectral type of the star (top panel) and $M_{d}$ vs. the stellar age (bottom panel) for a wide sample of HAeBe stars. Data have been taken from the compilation by Natta et al. (2000) (open symbols) and the results reported by Fuente et al. (2001) and this Letter (filled symbols). Filled circles indicate detections, while triangles indicate upper limits. Note that disk masses in HBe stars are at least an order of magnitude lower than in HAe stars.

system $\left(\sim 0.0013 M_{\odot}\right)$. Thus, planet formation should occur very fast, for planetary systems to exist at all around massive stars.

This Letter has been partially funded by the Spanish MCyT under projects DGES/AYA2000-927, ESP2001-4519-PE, and ESP2002-01693, and European FEDER funds. A. N. and L. T. acknowledge support from grant ARS-1/R/073/01 to the Osservatorio di Arcetri. A. R.-F. acknowledges support from ESP2002-01627 and AYA2002-10113-E.

\section{REFERENCES}

Beckwith, S. V. W., \& Sargent, A. I. 1991, ApJ, 381, 250

Brugel, E. W., Mundt, R., \& Buhrke, T. 1984, ApJ, 287, L73

Close, L. M., et al. 1997, ApJ, 489, 210

Curiel, S., Rodríguez, L. F., Cantó, J., Bohigas, J., Roth, M., \& Torrelles, J. M. 1989, Astrophys. Lett. Commun., 27, 299

Di Francesco, J., Evans, N. J., II, Harvey, P. M., Mundy, L. G., Guilloteau, S., \& Chandler, C. J. 1997, ApJ, 482, 433

Dullemond, C. P., van Zadelhoff, G. J., \& Natta, A. 2002, A\&A, 389, 464

Fich, M. 1993, ApJS, 86, 475

Fuente, A., Martín-Pintado, J., Bachiller, R., Neri, R., \& Palla, F. 1998, A\&A, 334,253

Fuente, A., Martín-Pintado, J., Bachiller, R., Rodríguez-Franco, A., \& Palla, F. 2002, A\&A, 387, 977
Fuente, A., Neri, R., Martín-Pintado, J., Bachiller, R., Rodríguez-Franco, A., \& Palla, F. 2001, A\&A, 366, 873

Girart, J. M., Curiel, S., Rodríguez, L. F., \& Cantó, L. 2002, Rev. Mexicana Astron. Astrofis., 38, 169

Leinert, C., Richichi, A., \& Haas, M. 1997, A\&A, 318, 472

Natta, A., Grinin, V. P., \& Mannings, V. 2000, in Protostars and Planets IV, ed. V. Mannings, A. Boss, \& S. S. Russell (Tucson: Univ. Arizona Press), 559

Polomski, E. F., Telesco, C. M., Piña R., \& Schulz, B. 2002, AJ, 124, 2207 Skinner, S. L., Brown, A., \& Stewart, R. T. 1993, ApJS, 87, 217

Testi, L., Natta, A., Shepherd, D. S., \& Wilner, D. J. 2003, A\&A, 403, 323

Thé, P. S., de Winter, D., \& Pérez, M. R. 1994, A\&AS, 104, 315 